\title{
Vitamina D e dialisi peritoneale. Non solo controllo dell'iperparatiroidismo
}

\author{
Roberto Russo
}

\author{
U.O. di Nefrologia Universitaria, Azienda Universitaria Consorziale Policlinico, Bari
}

\begin{abstract}
VITAMIN D AND PERITONEAL DIALYSIS: NOT ONLY AGAINST HYPERPARATHYROIDISM
Abstract. Vitamin D deficiency is highly prevalent in peritoneal dialysis (PD) patients. The administration of active vitamin D, or vitamin D analogs, in PD may have significant pleiotropic effects. Specifically, the improvement of the bactericidal response could decrease the risk of peritonitis, the antiproteinuric effect could preserve the residual renal function, the reduction of the peritoneal protein loss could decrease the risk of malnutrition, and the antifibrotic effect could reduce the risk of sclerosing encapsulating peritonitis.
\end{abstract}

Key words: Vitamin D, Peritoneal dialysis, Pleiotropic effects

Conflict of interest: None.

Financial support: None.

Accettato: 28 Novembre 2014

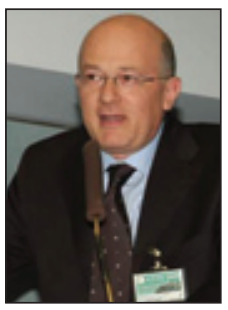

La carenza di vitamina $\mathrm{D}$ è frequente in dialisi. I pazienti in dialisi peritoneale (PD) presentano livelli di $25-\mathrm{OHD}_{3}$ più bassi rispetto ai pazienti in emodialisi e la perdita di vitamina $\mathrm{D}$ con il dialisato effluente rappresenta una delle cause principali. La vitamina D attiva e gli analoghi sintetici sono efficaci nel ridurre i livelli sierici di PTH. Rimane aperta la questione se i cosiddetti effetti pleiotropici della vitamina $\mathrm{D}$ possano essere significativi in PD.

L'incidenza di peritonite, la maggiore complicanza infettiva della $\mathrm{PD}$, sembra ridursi nei pazienti che assumono vitamina $\mathrm{D}$ attiva per via orale. Kerschbaum et al. (1) hanno raccolto i dati di 726 pazienti in PD e hanno rilevato, all'analisi multivariata, che il rischio di peritonite si riduce del $57 \%$ nei pazienti trattati con vitamina $\mathrm{D}$. La spiegazione può risiedere nell'effetto immunomodulatore della vitamina $\mathrm{D}$. Questa vitamina, infatti, svolge un ruolo nella funzione antimicrobica dei macrofagi isolati dai pazienti in PD (2). I macrofagi peritoneali trattati con 1-25 di-idrossicolecalciferolo aumentano la produzione di superossido e l'attività battericida, mentre l'attivazione del peptide antimicrobico intracellulare catelicidina, nei monociti e nei macrofagi, è vitamina D-dipendente (3). Rudnicki et al. (4) hanno esaminato gli effetti dei differenti dosaggi orali di calcitriolo sulla riduzione del rischio di peritonite, non trovando differenze tra i pazienti che assumono $0.25 \mathrm{mcg}$ o più rispetto a quelli che ricevono meno di $0.25 \mathrm{mcg}$. Il potenziamento dell'effetto battericida osservato da Rudnicki non è dose-dipendente.
L'effetto della vitamina D sulla riduzione della proteinuria nei pazienti con malattia renale cronica è stato ampiamente dimostrato. Coronel et al. riportano una riduzione della proteinuria nei pazienti in PD trattati con paracalcitolo (5). In uno studio successivo, gli stessi Autori rilevano che la riduzione della proteinuria $(21.9 \%)$ è associata a una riduzione delle perdite proteiche peritoneali (15.4\%) e a un aumento dell'ultrafiltrazione peritoneale (15.8\%). Questi interessanti dati non sono associati a un cambio della permeabilità peritoneale, così come dimostrato dall'assenza di modifiche del D/P della creatinina (6). Recentemente, Sánchez-Álvarez et al. hanno dimostrato che un trattamento di 6 mesi con paracalcitolo si associa a una riduzione della proteinuria del $20 \%$, ma, differentemente da quanto sostenuto da Coronel, non si associa a variazioni della perdita proteica peritoneale (7). Gli effetti antiinfiammatori e di modulazione del sistema del RAAS, descritti per il paracalcitolo, potrebbero esserne i responsabili.

Il trattamento prolungato con la dialisi peritoneale causa modificazioni morfologiche e funzionali del peritoneo, che includono una marcata fibrosi peritoneale e un massivo accumulo di collagene nella zona submesoteliale. Braun et al. (8) hanno analizzato 72 biopsie peritoneali trovando un'elevata espressione di recettori per la vitamina $\mathrm{D}$ nel peritoneo di pazienti con peritonite sclerosante incapsulante (SEP). Il trattamento con 22-oxacalcitriolo, un analogo della vitamina $\mathrm{D}$, sopprime la progressione dell'ispessimento peritoneale e del deposito di collagene in un modello murino di fibrosi peritoneale (9). L'effetto antifibrotico del 22-oxacalcitriolo è dose-dipendente ed è associato ad alcuni effetti collaterali come l'ipercalcemia. I miofibroblasti giocano un ruolo centrale nella fibrosi peritone- 
TABELLA I - EFFETTI PLEIOTROPICI POTENZIALI DELLA VITAMINA D IN DIALISI PERITONEALE

\author{
Effetto antifibrotico \\ - $\downarrow$ rischio di peritonite sclerosante \\ Riduzione della perdita proteica \\ - $\downarrow$ rischio di malnutrizione \\ Effetto battericida \\ - $\downarrow$ rischio di peritonite \\ Effetto antiproteinurico \\ - conservazione della funzione renale residua
}

ale e il 22-oxacalcitriolo potrebbe agire riducendo il numero dei miofibroblasti $\alpha$-SMA-positivi, che sono i maggiori produttori peritoneali di collagene (10). Infine, Kang et al. hanno valutato la capacità del paracalcitolo di prevenire la transizione epitelio-mesenchimale (EMT) (11). Il paracalcitolo riduce, in vitro, da parte di cellule mesoteliali umane in coltura, l'espressione dei marker di EMT, attraverso la soppressione del TGF- $\beta 1 /$ Smad signaling, mentre, nel ratto, in un modello sperimentale di peritonite sclerosante, riduce lo spessore peritoneale e la fibrosi.

Gli effetti pleiotropici della vitamina $\mathrm{D}$, in $\mathrm{PD}$, potrebbero migliorare la sopravvivenza dei pazienti e della tecnica dialitica. In particolare, il potenziamento dell'azione battericida potrebbe ridurre il rischio di peritonite, l'effetto antiproteinurico potrebbe preservare la funzione renale residua, la riduzione della perdita proteica peritoneale potrebbe ridurre il rischio di malnutrizione e l'effetto antifibrotico potrebbe ridurre il rischio di peritonite sclerosante incapsulante (Tab. I). Trial clinici sono necessari per confermare queste ipotesi suggestive.

\section{Riassunto}

Il deficit di vitamina $\mathrm{D}$ è molto frequente nei pazienti in dialisi peritoneale (PD). La somministrazione di vitamina D attiva o di analoghi potrebbe avere degli effetti pleiotropici significativi nei pazienti in PD. In particolare, un potenziamento dell'attività battericida potrebbe ridurre il rischio di peritonite, l'effetto antiproteinurico potrebbe mantenere la funzione renale residua, la riduzione della perdita proteica peritoneale potrebbe ridurre il rischio di malnutrizione e l'effetto antifibrotico potrebbe ridurre il rischio di peritonite sclerosante incapsulante.

Parole chiave: Vitamina D, Dialisi peritoneale, Effetti pleiotropici

Dichiarazione di conflitto di interesse: L'Autore dichiara di non avere conflitto di interessi.

Contributi economici agli Autori: L'Autore dichiara di non avere ricevuto sponsorizzazioni economiche per la preparazione dell'articolo.

\author{
Indirizzo dell'Autore: \\ Dr. Roberto Russo \\ U.O. di Nefrologia \\ Azienda Ospedaliero-Universitaria \\ Consorziale Policlinico di Bari \\ Piazza G. Cesare 11 \\ 70124 Bari \\ roberto.russo@policlinico.ba.it
}

\section{Bibliografia}

1. Kerschbaum J, Vychytil A, Lhotta K, et al. Treatment with oral active vitamin D is associated with decreased risk of peritonitis and improved survival in patients on peritoneal dialysis. Plos One 2013; 8: e67836.

2. Cohen ML, Douvdevani A, Chaimovitz C, Shany S. Regulation of TNF- $\alpha$ by $1 \alpha, 25$-dihydroxyvitamin D3 in human macrophages from CAPD patients. Kidney Int 2001; 59: 69-75.

3. Liu PT, Stenger S, Li H, et al. Toll-like receptor triggering of a vitamin D-mediated human antimicrobial response. Science 2006; 311: 1770-3.

4. Rudnicki M, Kerschbaum J, Hausdorfer J, Mayer G, Konig P. Risk factors for peritoneal dialysis-associated peritonitis: the role of oral active vitamin D. Perit Dial Int 2010; 30: 541-8.

5. Coronel F, Rodriguez-Cubillo B, Cigarran S, Gomis A. Effects of paricalcitol on hyperparathyroidism and proteinuria in peritoneal dialysis patients. Adv Perit Dial 2011; 27: 130-3.

6. Coronel F, Cigarran S, Gomis A, et al. Changes in peritoneal membrane permeability and proteinuria in patients on peritoneal dialysis after treatment with paricalcitol - a preliminary study. Clin Nephrol 2012; 78: 93-9.

7. Sánchez-Álvarez JE, Rodríguez-Suárez C, Coronel-Aguilar D, et al. Paricalcitol reduces proteinuria but does not modify peritoneal protein loss in patients on peritoneal dialysis. Nefrologia 2013; 33: 70-6.

8. Braun N, Fritz P, Biegger D, et al. Difference in the expression of hormone receptors and fibrotic markers in the human peritoneum-Implications for therapeutic targets to prevent encapsulating peritoneal sclerosis. Perit Dial Int 2011; 31: 291-300.

9. Hirose M, Nishino T, Obata Y, et al. 22-Oxacalcitriol prevents progression of peritoneal fibrosis in a mouse model. Perit Dial Int 2013; 33 (2): 132-42.

10. Li Y, Spataro BC, Yang J, Dai C, Liu Y. 1,25-Dihydroxyvitamin $\mathrm{D}$ inhibits renal interstitial myofibroblast activation by inducing hepatocyte growth factor expression. Kidney Int 2005; 68: 1500-10.

11. Kang SH, Kim SO, Cho KH, Park JW, Yoon KW, Do JY. Paricalcitol ameliorates epithelial-to-mesenchymal transition in the peritoneal mesothelium. Nephron Exp Nephrol 2014; 126: 1-7. 